\title{
Computational Analysis of Theacrine, a Purported Nootropic and Energy-Enhancing Nutritional Supplement
}

\author{
Bradley 0. Ashburn*, Diana J. Le, Corin K. Nishimura \\ Mathematics and Natural Sciences Division, University of Hawaii-Leeward Community College, Pearl City, HI, USA \\ Email: *bashburn@hawaii.edu
}

How to cite this paper: Ashburn, B.O., Le, D.J. and Nishimura, C.K. (2019) Computational Analysis of Theacrine, a Purported Nootropic and Energy-Enhancing Nutritional Supplement. Computational Chemistry, 7, 27-37.

https://doi.org/10.4236/cc.2019.71002

Received: November 22, 2018

Accepted: December 25, 2018

Published: December 28, 2018

Copyright $\odot 2019$ by authors and Scientific Research Publishing Inc. This work is licensed under the Creative Commons Attribution International License (CC BY 4.0).

http://creativecommons.org/licenses/by/4.0/

\section{(c) (i) Open Access}

\begin{abstract}
Herein is the first reported conceptual density functional theory (DFT) investigation of the purine alkaloid theacrine and the comparison of quantum chemical properties to the closely related stimulant caffeine. DFT global chemical reactivity descriptors (chemical hardness/softness, chemical potential/electronegativity, and electrophilicity) and local reactivity descriptors (Fukui functions and dual descriptor) were calculated for both compounds using Spartan '16 software. All calculations were carried out at the B3LYP/6-31G* level of theory. Reactivity analysis of the Fukui dual descriptor calculations reveals sites of nucleophilic and electrophilic attack. The results provide a solid chemical foundation for understanding how theacrine interacts with cellular systems.
\end{abstract}

\section{Keywords}

Theacrine, Caffeine, Density Functional Theory, Computational Analysis

\section{Introduction}

Caffeine and other dietary supplements advertised to increase energy and alertness are widely used by the general population as well as those involved in physically and cognitively demanding tasks [1] [2] [3]. Caffeine is consumed by $89 \%$ of adults in the US [4], and $85 \%$ of the US population consumes at least one caffeinated drink per day [5]. As an ergogenic aid, caffeine is used by athletes, military personnel, and others who seek improved physical and mental performance [1] [3] [6]. Caffeine is widely available, commonly found both naturally occurring and as an additive in foods, drinks and dietary supplements. Pharmacological studies show the mechanism of action as an adenosine blocker in the brain resulting in inhibitory effects to the CNS [7]. Since caffeine has a similar struc- 
ture to adenosine, a hormone that has sleep-promoting effects, caffeine has the potential to bind to adenosine receptor sites, thus preventing adenosine reuptake that triggers tiredness [7]. The result is increased alertness and enhanced ability to maintain attention.

More recently, supplements containing theacrine, a purine alkaloid found naturally in the leaves of the Camellia Kucha plant, has gained popularity among those seeking to increase energy and to improve focus, concentration, and mood. Though not as extensively studied as caffeine, early evidence does support the efficacy of theacrine-containing supplements in improving subjective measures related to energy, mood, and fatigue reduction [8] [9]. Safety data with theacrine supplementation indicates when taken as directed, the compound is safe and not shown to adversely affect physiological indicators such as heart rate, blood pressure, rate pressure product, or other clinical safety measures [9] [10]. Theacrine-containing products are widely available, revealing the growing popularity of this dietary supplement. Given the widespread use of caffeine and other energy supplements, it is likely theacrine will continue to gain in popularity.

Density Functional Theory (DFT) enables scientists to elucidate valuable descriptions of chemical reactivity, molecular structure, vibrational frequencies, and the energetics of chemical reactions [11] [12] [13] [14] [15]. DFT has produced powerful tools for the study of organic reactivity via the use of theoretical reactivity indices on a global and local scale. DFT is widely utilized by researchers in chemistry, physics and materials science due to the development of accurate density functionals.

Previous DFT studies on caffeine have yielded valuable insights into its chemical reactivity [16] [17] but no such studies have been performed on theacrine. The goal of this computational analysis is to investigate the electronic properties of theacrine to predict its relative stability and reactivity compared to caffeine.

\section{Materials and Methods}

\subsection{Computational Details}

Quantum chemical calculations were performed using Spartan '16 computational software on a Macintosh computer. Geometry optimization and single point energy calculations were carried out at the B3LYP/6-31G* level of theory and verified by the absence of any imaginary frequencies.

\subsection{Global Quantum Chemical Descriptors}

\subsubsection{Electronic Chemical Potential $(\mu)$ and Mulliken Electronegativity} $(\chi)$

The electronic chemical potential $(\mu)$ of a molecule is the change in energy with respect to the number of electrons $(\mathrm{N})$ at a fixed external potential $v(\mathrm{r})$ [18] [19]. This represents the tendency of electrons to escape from an atom or molecule.

$$
\mu=\left(\frac{\partial E}{\partial N}\right)_{v(r)}
$$


Application of the finite difference approximation yields the simplified expression:

$$
\mu \approx-\frac{(I P+E A)}{2}
$$

where $I P$ represents the first ionization potential and $E A$ represents the electron affinity of an atom or molecule. The Koopman theorem [20] and Kohn-Sham formalism [12] show that the ionization potential (IP) can be approximated by the energy of the highest occupied molecular orbital $\left(-E_{\text {Номо }}\right)$ and the electron affinity $(E A)$ can be approximated by the energy of the lowest unoccupied molecular orbital $\left(-E_{L U M O}\right)$.

$$
\mu \approx \frac{\left(E_{\text {HОMO }}+E_{\text {LUMO }}\right)}{2}
$$

Given that electronegativity $(\chi)$ is characterized as the tendency of an atom to attract electrons, it can be written as the negative of the chemical potential $(\mu)$ :

$$
\chi=-\mu
$$

\subsubsection{Chemical Hardness $(\eta)$ and Softness $(S)$}

The hard and soft acids and bases (HSAB) principle presented by Pearson [21] [22] [23] states that Lewis acids can be classified as either hard or soft [24] and that the most favorable interactions in an acid/base reaction occur between hard/hard or soft/soft pairs. It would thus be beneficial to have a metric that measures the hardness/softness of molecules. Parr defined a quantitative expression for chemical hardness $(\eta)$ as the change in chemical potential $(\mu)$ of a molecule with respect to the number of electrons $(\mathrm{N})$ at a fixed external potential $v(\mathrm{r})$. This represents the resistance of a molecule to exchange electron density with the environment.

$$
\eta=\left(\frac{\partial \mu}{\partial N}\right)_{v(r)}
$$

Using the finite difference approximation and substituting the ionization potential $(I P)$ for $-E_{\text {HOMO }}$ and electron affinity $(E A)$ for $-E_{L U M O}$ the chemical hardness can be expressed as:

$$
\eta \approx \frac{(I P-E A)}{2} \approx \frac{\left(E_{L U M O}-E_{H O M O}\right)}{2}
$$

Chemical softness $(S)$ is the reciprocal of chemical hardness $(\eta)$ :

$$
S=\frac{1}{\eta}
$$

\subsubsection{Electrophilicity Index $(\omega)$}

Maynard [25] and Parr [26] defined the electrophilicity index $(\omega)$ to represent the tendency of an electrophile to acquire more electron density (chemical potential) divided by the resistance to exchange electron density with the environment (chemical hardness). It is a quantitative value intrinsic to the molecule and 
has become a powerful tool to predict reactivity. One can consider electrophilicity to represent the stabilization energy of a system when it gains electron density from the environment.

$$
\omega=\frac{\mu^{2}}{2 \eta}
$$

\subsection{Local Quantum Chemical Descriptors}

\section{Fukui Functions and Dual Descriptor}

The Fukui function $f(r)$ represents the change in electron density at point $r$ with respect to the change in the number of electrons $N$ at a fixed external potential $v(r)$ [27]:

$$
f(r)=\left(\frac{\partial \rho(r)}{\partial N}\right)_{\nu(r)}
$$

Parr expanded the use of the Fukui function to show localized areas of nucleophilicity $\left(f^{+}\right)$and electrophilicity $\left(f^{-}\right)$called the condensed Fukui functions [28] [29].

$$
f^{+}(r) \approx \rho_{(N+1)}(r)-\rho_{N}(r)
$$

and

$$
f^{-}(r) \approx \rho_{N}(r)-\rho_{N-1}(r)
$$

where $\rho_{N}, \rho_{N-1}$, and $\rho_{(N+1)}$ are the atomic charges in the neutral, anionic and cationic species, respectively.

It has been discovered that combining the two functions provides the most useful description in what is known as the dual descriptor [30] [31] [32]. Where $\Delta f_{k}(r)<0$ represents an atom $k$ of a molecule that is nucleophilic and $\Delta f_{k}(r)>0$ an atom $\mathrm{k}$ that is electrophilic.

$$
\Delta f(r)=\left(\frac{\partial f(r)}{\partial N}\right)_{v(r)}
$$

A condensed form is represented by:

$$
\Delta f(r)=f_{k}^{+}-f_{k}^{-}
$$

\section{Results and Discussion}

Full unconstrained molecular geometry optimizations of theacrine and caffeine were carried out using Wavefunction Spartan '16 software [33] using the exchange correlation functional by Becke [34] with gradient-corrected correlation provided by Lee, Yang and Parr [35] with the $6-31 G^{*}$ basis set. This DFT method was chosen since it is fast, less computationally intensive, takes electron correlation into account, and provides accuracy in reproducing experimental data [36]. Figure 1 depicts the optimized molecular structures of the most stable forms of theacrine and caffeine. Optimized geometries were confirmed by vibrational analysis calculations and characterized as minima in their potential energy 
surface via the absence of an imaginary frequency.

Visualization of the contour plots of the Highest Occupied Molecular Orbital (HOMO) and Lowest Unoccupied Molecular Orbital (LUMO) are shown in Figure 2. Analysis of the frontier molecular orbitals provides valuable information regarding nodal patterns and individual atom contributions. Extended conjugation in the HOMO of caffeine with $\mathrm{C} 1, \mathrm{C} 2, \mathrm{C} 4$ and $\mathrm{N} 4$ is observed compared to the more localized conjugation of the HOMO of theacrine with $\mathrm{C} 1, \mathrm{C} 2$ and $\mathrm{C} 4$. This is rationalized by resonance of $\mathrm{N} 4$ with the adjacent carbonyl group. This localization may be a factor in the Fukui dual descriptor results which predict the reactive sites of each molecule. The HOMO-LUMO energy gap for theacrine is $4.73 \mathrm{eV}$ compared to $5.12 \mathrm{eV}$ for caffeine.

Table 1 shows the global chemical reactivity descriptors. Theacrine has an electronic chemical potential $(\mu)$ of $-3.10 \mathrm{eV}$ compared to $-3.41 \mathrm{eV}$ in caffeine which shows that electrons will have a greater escaping tendency in theacrine, thus indicating theacrine to be a more reactive and less stable molecule compared to caffeine.

Table 1. Global chemical reactivity indices of theacrine and caffeine.

\begin{tabular}{ccc}
\hline Reactivity & \multicolumn{2}{c}{$\mathrm{B}_{\mathrm{L}} \mathrm{LYP} / 6-31 \mathrm{G}^{*}$} \\
\cline { 2 - 3 } Index & theacrine & caffeine \\
\hline $\mathrm{E}_{\text {HOмо }}(\mathrm{eV})$ & -5.46 & -5.97 \\
$\mathrm{E}_{\mathrm{LUMO}}(\mathrm{eV})$ & -0.73 & -0.85 \\
$\mathrm{E}_{\mathrm{Gap}}(\mathrm{eV})$ & 4.73 & 5.12 \\
$\mu(\mathrm{eV})$ & -3.10 & -3.41 \\
$\chi(\mathrm{eV})$ & 3.10 & 3.41 \\
$\eta(\mathrm{eV})$ & 2.37 & 2.56 \\
$S(\mathrm{eV})$ & 0.422 & 0.391 \\
$\omega(\mathrm{eV})$ & 2.03 & 2.27 \\
$\log P$ & -1.61 & -1.12 \\
\hline
\end{tabular}

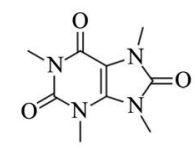

theacrine (1)

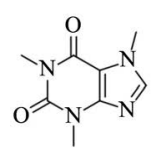

caffeine (2)
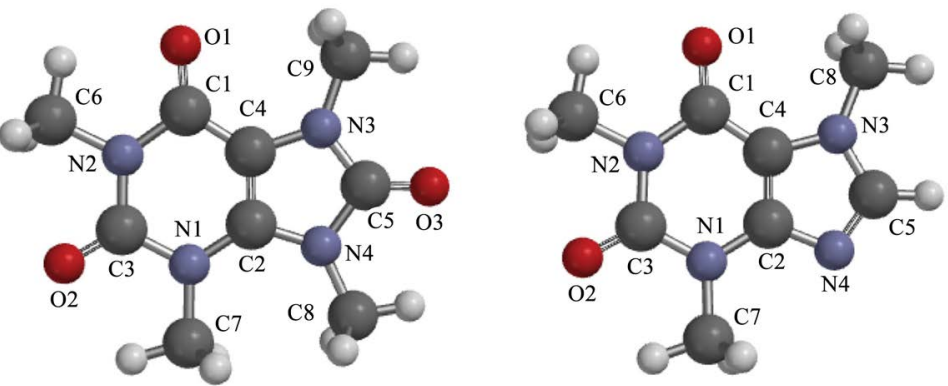

Figure 1. The 2D and 3D structures of theacrine (1) and caffeine (2). 


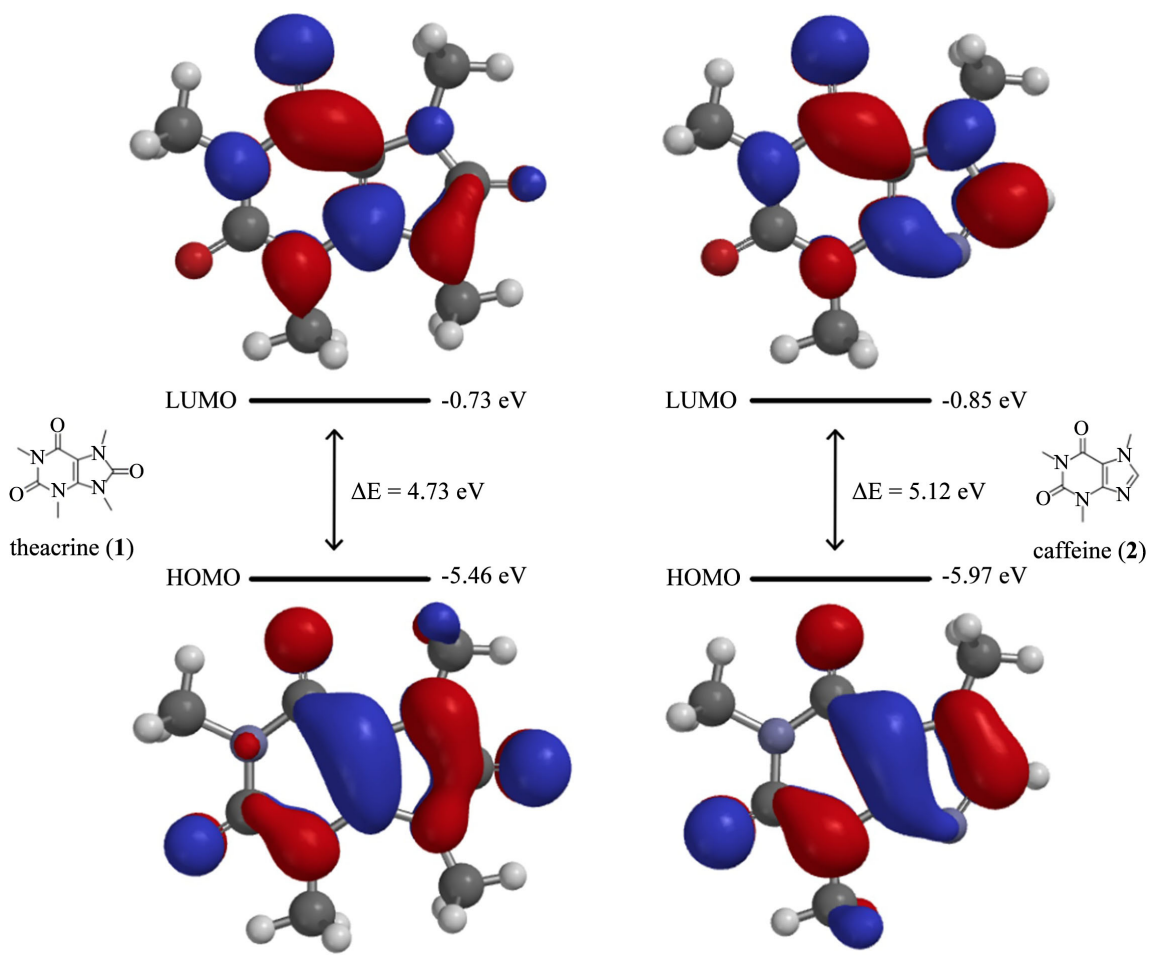

Figure 2. Frontier Molecular Orbitals of theacrine and caffeine.

Chemical hardness $(\eta)$ is also associated with the stability and reactivity of a molecule since it measures the resistance to change in the electron distribution or charge transfer. Theacrine is less hard and thus more reactive than caffeine. Chemical softness $(S)$ is the measure of how readily the molecule exchanges electron density with the environment. Since theacrine is softer than caffeine, it has more ability to react. But another factor to consider is the higher electrophilicity $(\omega)$ value for caffeine compared to theacrine which means that caffeine has a greater propensity to accept electrons and is thus a stronger electrophile.

The partition coefficient of a compound is a widely used metric for determining lipophilicity, which is useful for identifying compounds which may have similar likeness to drugs and possible interaction with biological macromolecules such as receptors and enzymes [37]. Lipophilicity is an important physicochemical property of a potential drug since it affects solubility, absorption, membrane penetration, distribution, and excretion. The partition coefficient is the quotient of the concentration of the compound in a lipophilic solvent (typically 1-octanol) and the concentration of the compound in water. The logarithm of the partition coefficient is referred to as $\log \mathrm{P}$. A positive value for $\log \mathrm{P}$ means the compound is more lipophilic, whereas a negative value means the compound is more hydrophilic. The data shows that while both compounds are hydrophilic, theacrine hydrophilicity is greater than that of caffeine. This can be rationalized by the extra carbonyl group of theacrine.

Table 2 and Table 3 show the natural bond orbital charge, $\mathrm{f}^{+}$value, $\mathrm{f}^{-}$value, and the dual descriptor $(\Delta f)$ value calculated for every atom in theacrine and 
caffeine except for the hydrogens. The natural bond orbital charges were used in Equations (10) and (11) to determine the $f^{+}$and $f^{-}$Fukui indices respectively. The combined values according to Equation (13) yields the dual descriptor, where $\Delta f$ is a function that represents the reactivity of the atoms in each molecule; positive values represent sites of electrophilic attack and negative values represent sites of nucleophilic attack.

Table 2. Fukui indices for theacrine.

\begin{tabular}{ccccc}
\hline atom & charge & $f_{k}^{+}$ & $f_{k}^{-}$ & $\Delta f$ \\
\hline C1 & +0.634 & -0.153 & 0.008 & -0.161 \\
C2 & +0.469 & -0.2 & -0.064 & -0.136 \\
C3 & +0.837 & 0.001 & 0 & 0.001 \\
N1 & -0.408 & -0.018 & -0.053 & 0.035 \\
N2 & -0.480 & -0.022 & -0.013 & -0.009 \\
C4 & +0.124 & -0.022 & -0.141 & 0.119 \\
N3 & -0.348 & -0.013 & -0.086 & 0.073 \\
C5 & +0.826 & -0.016 & -0.009 & -0.007 \\
N4 & -0.426 & -0.013 & -0.034 & 0.021 \\
O1 & -0.510 & -0.135 & -0.106 & -0.029 \\
O2 & -0.536 & -0.066 & -0.100 & 0.034 \\
O3 & -0.482 & -0.068 & -0.166 & 0.098 \\
C6 & -0.480 & 0.011 & 0.009 & 0.002 \\
C7 & -0.491 & 0.014 & 0.014 & 0 \\
C8 & -0.490 & 0.015 & 0.014 & 0.001 \\
C9 & -0.484 & 0.013 & 0.019 & -0.006 \\
\hline & & & &
\end{tabular}

Table 3. Fukui indices for caffeine.

\begin{tabular}{ccccc}
\hline atom & charge & $f_{k}^{+}$ & $f_{k}^{-}$ & $\Delta f$ \\
\hline C1 & +0.649 & -0.148 & 0.015 & -0.163 \\
C2 & +0.362 & -0.098 & -0.037 & -0.061 \\
C3 & +0.837 & -0.002 & 0.009 & -0.011 \\
N1 & -0.453 & -0.019 & -0.136 & 0.117 \\
N2 & -0.497 & -0.018 & -0.014 & -0.004 \\
C4 & -0.027 & -0.017 & -0.172 & 0.155 \\
N3 & -0.363 & -0.055 & -0.026 & -0.029 \\
C5 & +0.229 & -0.164 & -0.091 & -0.073 \\
N4 & -0.521 & -0.039 & -0.043 & 0.004 \\
O1 & -0.620 & -0.127 & -0.119 & -0.008 \\
O1 & -0.636 & -0.067 & -0.142 & 0.075 \\
C6 & -0.472 & 0.012 & 0.010 & 0.002 \\
C7 & -0.470 & 0.013 & 0.019 & -0.006 \\
C8 & -0.463 & 0.019 & 0.018 & 0.001 \\
\hline
\end{tabular}


For theacrine, the calculations show positive values of the $\Delta f$ at $\mathrm{C} 4, \mathrm{O} 3, \mathrm{~N} 3$, $\mathrm{N} 1, \mathrm{O} 2$, and N4. Electrophilic attack has the highest likelihood on these atoms. It is interesting to see the highest value on C4 and not one of the heteroatoms. That could provide insight into preferential reactivity. The two notable negative values of the $\Delta f$ are at $\mathrm{C} 1$ and $\mathrm{C} 2$ where nucleophilic attack could occur. Since $\mathrm{C} 1$ and $\mathrm{C} 2$ are part of the conjugated system, the calculated values make sense. For caffeine, notable positive values of $\Delta f$ are found on $\mathrm{C} 4, \mathrm{~N} 1$, and O1. C4 is again the most likely site for electrophilic attack. Notable negative values of the $\Delta f$ are found at $\mathrm{C} 1, \mathrm{C} 5$, and $\mathrm{C} 2$. All three of these atoms are involved in the conjugated system with the C1-O1 carbonyl which would be predicted to be susceptible to nucleophilic attack. A major difference in reactivity between theacrine and caffeine is seen at C5, where in caffeine it is predicted to be a site for nucleophilic attack but that is not the case for C5 in theacrine. This is rationalized by the resonance stabilization of the carbonyl carbon in the urea moiety of theacrine.

\section{Conclusion}

Theacrine is more reactive than caffeine as judged by electronic chemical potential and chemical hardness and softness. Caffeine, however, was found to be more electrophilic. Theacrine has a more negative $\log \mathrm{P}$ value which means that it is more hydrophilic. This reduced lipophilicity could result in more difficulty diffusing through cellular membranes and the blood-brain barrier. Fukui dual descriptor analysis shows $\mathrm{C} 4$ of both theacrine and caffeine to be the most likely site of electrophilic attack and $\mathrm{C} 1$ and $\mathrm{C} 2$ of both molecules to be sites of nucleophilic attack. The goal of the study was achieved by uncovering the electronic properties and reactivity metrics of theacrine in comparison to caffeine. Using the data gathered from this study, further analyses, such as molecular docking, can be undertaken to further understand how theacrine interacts with cellular systems.

\section{Acknowledgements}

The authors would like to thank Leeward Community College for providing the computer software and hardware necessary to conduct this computational analysis.

\section{Conflicts of Interest}

The authors declare no conflicts of interest regarding the publication of this paper.

\section{References}

[1] Lieberman, H.R., Marriott, B.P., Williams, C., Judelson, D.A., Glickman, E.L., Geiselman P.J., Dotson, L. and Mahoney, C.R. (2015) Patterns of Dietary Supplement Use among College Students. Clinical Nutrition, 34, 976-985. https://doi.org/10.1016/j.clnu.2014.10.010 
[2] Dickinson, A., Blatman, J., El-Dash, N. and Franco, J.C. (2014) Consumer Usage and Reasons for Using Dietary Supplements: Report of a Series of Surveys. Journal of the American College of Nutrition, 33, 176-182. https://doi.org/10.1080/07315724.2013.875423

[3] McLellan, T.M., Riviere, L.A., Williams, K.W., McGurk, D. and Lieberman, H.R. (2018) Caffeine and Energy Drink Use by Combat Arms Soldiers in Afghanistan as a Countermeasure for Sleep Loss and High Operational Demands. Nutritional Neuroscience. https://doi.org/10.1080/1028415X.2018.1443996

[4] Fulgoni III, V.L., Keast, D.P. and Lieberman, H.R. (2015) Trends in Intake and Sources of Caffeine in the Diets of US Adults: 2001-2010. The American Journal of Clinical Nutrition, 101, 1081-1087. https://doi.org/10.3945/ajcn.113.080077

[5] Mitchell, D.C., Knight, C.A., Hockenberry, J., Teplansky, R. and Hartman, T.J. (2014) Beverage Caffeine Intakes in the U.S. Food and Chemical Toxicology, 63, 136-142. https://doi.org/10.1016/j.fct.2013.10.042

[6] Del Coso, J., Muñoz, G. and Muñoz-Guerra, J. (2010) Prevalence of Caffeine Use in Elite Athletes Following Its Removal from the World Anti-Doping Agency List of Banned Substances. Applied Physiology, Nutrition, and Metabolism, 36, 555-561. https://doi.org/10.1139/h11-052

[7] Heckman, M.A., Weil, J. and Mejia, E.G.D. (2010) Caffeine (1,3,7-trimethylxanthine) in Foods: Comprehensive Review on Consumption, Functionality, Safety, and Regulatory Matters. Journal of Food Science, 75, 77-87. https://doi.org/10.1111/j.1750-3841.2010.01561.x

[8] Habowski, S.M., Sandrock, J.E., Kedia, A.W. and Ziegenfuss, T. (2014) The Effects of Teacrine, a Nature-Identical Purine Alkaloid, on Subjective Measures of Cognitive Function, Psychometric and Hemodynamic Indices in Healthy Humans: A Randomized, Double-Blinded Crossover Pilot Trial. Journal of the International Society of Sports Nutrition, 11, 49-50. https://doi.org/10.1186/1550-2783-11-S1-P49

[9] Kuhman, D.J., Joyner, K.J. and Bloomer, R.J. (2015) Cognitive Performance and Mood Following Ingestion of a Theacrine-Containing Dietary Supplement, Caffeine, or Placebo by Young Men and Women. Nutrients, 7, 9618-9632. https://doi.org/10.3390/nu7115484

[10] Taylor, L., Mumford, P., Roberts, M., Hayward, S., Mullins, J., Urbina, S. and Wilborn, C. (2016) Safety of TeaCrine ${ }^{\oplus}$, a Non-Habituating, Naturally-Occurring Purine Alkaloid over Eight Weeks of Continuous Use. Journal of the International Society of Sports Nutrition, 13, 1-14. https://doi.org/10.1186/s12970-016-0113-3

[11] Parr, R.G. and Yang, W. (1989) Density-Functional Theory of Atoms and Molecules, Oxford University Press, Oxford.

[12] Kohn, W. and Sham, L.J. (1965) Self-Consistent Equations Including Exchange and Correlation Effects. Physical Review B, 140, A1133-A1138. https://doi.org/10.1103/PhysRev.140.A1133

[13] Becke, A.D. (1997) Density-Functional Thermochemistry. V. Systematic Optimization of Exchange-Correlation Functionals. The Journal of Chemical Physics, 107, 8554-8560. https://doi.org/10.1063/1.475007

[14] Domingo, L.R., Rios-Gutierrez, M. and Perez, P. (2016) Applications of the Conceptual Density Functional Theory Indices to Organic Chemistry Reactivity. Molecules, 21, 748-770. https://doi.org/10.3390/molecules21060748

[15] Koch, W. and Holthausen, M.C. (2011) A Chemist's Guide to Density Functional Theory. 2nd Edition, Wiley-VCH.

[16] Salihovic, M., Huseinovic, S., Spirtovic-Halilovic, S., Osmanovic, A., Dedic, A., 
Ašrmovic, Z. and Završnik, D. (2014) DFT Study and Biological Activity of Some Methylxanthines. Journal of the Serbian Chemical Society, 42, 31-36.

[17] Kouakou, V., Niamien, P.M., Yapo, A.J., Diaby, S. and Trokourey, A. (2016) Experimental and DFT Studies on the Behavior of Caffeine as Effective Corrosion Inhibitor of Copper in $1 \mathrm{M} \mathrm{HNO}_{3}$. Orbital: The Electronic Journal of Chemistry, 8 , 66-79.

[18] Parr, R.G. and Pearson, R.G. (1983) Absolute Hardness: Companion Parameter to Absolute Electronegativity. Journal of the American Chemical Society, 105, 7512-7516. https://doi.org/10.1021/ja00364a005

[19] Parr, R.G. and Yang, W. (1989) Density Functional Theory of Atoms and Molecules. Oxford University Press, New York.

[20] Koopmans, T. (1933) Uber die Zuordnung von Wellenfunktionen und Eigenwerten zu den Einzelnen Elektronen Eines Atoms. Physica, 1, 104-113. https://doi.org/10.1016/S0031-8914(34)90011-2

[21] Pearson, R.G. and Songstad, J. (1967) Application of the Principle of Hard and Soft Acids and Bases to Organic Chemistry. Journal of the American Chemical Society, 89, 1827-1836. https://doi.org/10.1021/ja00984a014

[22] Pearson, R.G. (1973) Hard and Soft Acids and Bases. Dowden, Hutchinson and Ross, Stroudsberg.

[23] Pearson, R.G. (1997) Chemical Hardness: Applications from Molecules to Solids. Wiley-VCH Verlag GMBH, Weinheim. https://doi.org/10.1002/3527606173

[24] Yang, W. and Parr, R.G. (1985) Hardness, Softness, and the Fukui Function in the Electronic Theory of Metals and Catalysis. Proceedings of the National Academy of Sciences of the United States of America, 82, 6723-6726.

https://doi.org/10.1073/pnas.82.20.6723

[25] Maynard, A.T., Huang, M., Rice, W.G. and Covel, D.G. (1998) Reactivity of the HIV-1 Nucleocapsid Protein 77 Zinc Finger Domains from the Perspective of Density-Functional Theory. Proceedings of the National Academy of Sciences of the United States of America, 95, 11578-11583. https://doi.org/10.1073/pnas.95.20.11578

[26] Parr, R.G., von Szentpaly, L. and Liu, S. (1999) Electrophilicity Index. Journal of the American Chemical Society, 121, 1922-1924. https://doi.org/10.1021/ja983494x

[27] Fukui, K. (1982) Role of Frontier Orbitals in Chemical Reactions. Science, 218, 747-754. https://doi.org/10.1126/science.218.4574.747

[28] Parr, R.G. and Yang, W. (1984) Density Functional Approach to the Frontier-Electron Theory of Chemical Reactivity. Journal of the American Chemical Society, 106, 4049-4050. https://doi.org/10.1021/ja00326a036

[29] Yang, W., Parr, R.G. and Pucci, R. (1984) Electron Density, Kohn-Sham Frontier Orbitals, and Fukui Functions. The Journal of Chemical Physics, 81, 2862-2863. https://doi.org/10.1063/1.447964

[30] Morell, C., Grand, A. and Toro-Labbe, A. (2005) New Dual Descriptor for Chemical Reactivity. The Journal of Physical Chemistry A, 109, 205-212.

[31] Morell, C., Grand, A. and Toro-Labbe, A. (2006) New Theoretical Support for Using the $f(r)$ Descriptor. Chemical Physics Letters, 425, 342-346. https://doi.org/10.1016/j.cplett.2006.05.003

[32] Martinez-Araya, J.I. (2014) Why Is the Dual Descriptor a More Accurate Local Reactivity Descriptor than Fukui Functions? Journal of Mathematical Chemistry, 53, 451-465. https://doi.org/10.1007/s10910-014-0437-7 
[33] Shao, et al. (2006) Advances in Methods and Algorithms in a Modern Quantum Chemistry Program Package. Physical Chemistry Chemical Physics, 27, 3172-3191. https://doi.org/10.1039/B517914A

[34] Becke, A.D. (1988) Density-Functional Exchange-Energy Approximation with Correct Asymptotic Behavior. Physical Review A, 38, 3098-3100. https://doi.org/10.1103/PhysRevA.38.3098

[35] Lee, C., Yang, W. and Parr, R.G. (1988) Development of the Colle-Salvetti Correlation-Energy Formula into a Functional of the Electron Density. Physical Review B, 37, 785-789. https://doi.org/10.1103/PhysRevB.37.785

[36] Nkungli, N.K., Ghogomu, J.N., Nogheu, L.N. and Gadre, S.R. (2015) DFT and TD-DFT Study of Bis[2-(5-Amino-[1,3,4]-Oxadiazol-2-yl)Phenol](Diaqua)M(II) Complexes $[\mathrm{M}=\mathrm{Cu}, \mathrm{Ni}$ and $\mathrm{Zn}]$ : Electronic Structures, Properties and Analyses. Computational Chemistry, 3, 29-44. https://doi.org/10.4236/cc.2015.33005

[37] Leo, A., Hansch, C. and Elkins, D. (1971) Partition Coefficients and Their Uses. Chemical Reviews, 71, 525-616. https://doi.org/10.1021/cr60274a001 